\title{
Magnetofluidization of fine magnetite powder
}

\author{
J. M. Valverde, ${ }^{1}$ M. J. Espin, ${ }^{2}$ M. A. S. Quintanilla, ${ }^{1}$ and A. Castellanos ${ }^{1}$ \\ ${ }^{1}$ Department of Electronics and Electromagnetism, University of Seville, Avenida Reina Mercedes s/n, 41012 Sevilla, Spain \\ ${ }^{2}$ Department of Applied Physics II, University of Seville, Avenida Reina Mercedes s/n, 41012 Sevilla, Spain
}

(Received 16 December 2008; published 26 March 2009)

\begin{abstract}
The behavior of a fluidized bed of fine magnetite particles as affected by a cross-flow magnetic field is investigated. A distinct feature of this naturally cohesive powder, as compared to noncohesive magnetic grains usually employed in magnetofluidized beds, is that the fluidized bed displays a range of stable fluidization even in the absence of an external magnetic field. Upon application of the magnetic field, the interval of stable fluidization is extended to higher gas velocities and bed expansion is enhanced. We have measured the tensile strength as affected by application of the external magnetic field according to two different operation modes. In the $H$ off-on operation mode, the bed is driven to bubbling in the absence of external magnetic field. Once the gas velocity is decreased below the bubbling onset and the bed has returned to stable fluidization due to natural cohesive forces, the field is applied. In the $H$ on-on mode, the field is maintained during the whole process of bubbling and return to stable fluidization. It is found that the tensile strength of the naturally stabilized bed is not essentially changed by application of the field ( $H$ off-on) since the magnetic field cannot alter the bed structure once the particles are jammed in the stable fluidization state. Magnetic forces within the bulk of the jammed bed are partially canceled as a result of the anisotropic nature of the dipole-dipole interaction between the particles, which gives rise to just a small increment of the tensile strength. On the other hand, when the field is held on during bubbling and transition to stable fluidization ( $H$ on-on mode), the tensile strength is appreciably increased. This suggests the formation of particle chains when the particles are not constrained due to the dipole-dipole attractive interaction which affects the mechanical strength of the stably fluidized bed. Experimental data are analyzed in the light of theoretical models on magnetic surface stresses.
\end{abstract}

DOI: 10.1103/PhysRevE.79.031306

PACS number(s): 81.05.Rm, 47.55.Lm, 61.43.Gt, 47.65.-d

\section{INTRODUCTION}

Gas-solid fluidization technology is used in many industrial applications by virtue of its high gas-solid contact efficiency. Fluidization is accomplished by passing a gas through a vertically oriented bed of granular material. At a certain superficial gas velocity $v_{g}=v_{\mathrm{mf}}$ the drag balances the material weight per unit area and the grains become fluidized in a usually heterogeneous state. A major concern that besets fluidized bed reactors lies in the bypassing of gas through bubbles, which lowers contact efficiency and leads to uncertainties in process scaleup [1]. Bubbling fluidization is the common behavior found in fluidized beds of coarse granules (typically of size $d_{p} \gtrsim 100 \mu \mathrm{m}$ ) as soon as the gas velocity surpasses the minimum fluidization velocity $v_{\mathrm{mf}}$. This type of behavior is the so-called Geldart B behavior according to Geldart's diagram [2], which was originally derived from empirical observations on beds fluidized by air at ambient conditions. For intermediate-size particles $\left(d_{p}\right.$ typically between 20 and $50 \mu \mathrm{m}$ ), the van der Waals attractive forces between the particles become comparable to particle weight and are capable of suppressing bubbles in an interval of gas velocities between $v_{\mathrm{mf}}$ and $v_{g}=v_{c}>v_{\mathrm{mf}}$, where $v_{c}$ is the gas velocity at bubbling instability (Geldart A behavior [2]). Interparticle attractive forces may indeed provide the fluidized bed with an effective elastic modulus that stabilizes it against small disturbances $[3,4]$. In the stable state, interparticle contacts are permanently held by the attractive forces; the bed is jammed and takes the appearance of a weak solid [5]. Fluidlike behavior is accompanied by instability to bubbling when the gas velocity just equals $v_{c}$. When particle size is de- creased below about $20 \mu \mathrm{m}$, natural van der Waals forces between the particles are exceedingly large as compared to particle weight. Consequently, these powders, when fluidized, tend to rise as a slug of solids or to form channels through which the fluid escapes rather than being distributed through the bulk (Geldart $\mathrm{C}$ behavior [2]). It has been shown, however, that certain preconditioned fine powders with flow additives (of particle size around $10 \mu \mathrm{m}$ ) may display a nonbubbling fluidlike behavior [6], albeit this is not a truly stable state since local bubbles, which do not reach a macroscopic size, are seen to be continuously developed [7].

Artificial enhancement of interparticle forces has been investigated as a potential tool to suppress the growth of gas bubbles in the fluidization of Geldart's B coarse granular materials. It has been reported that enhancing the interparticle force between fluidized granules may effectively shift their fluidization behavior from bubbling to stable fluidization. For example, bubbling beds can be stabilized by incremental addition of a liquid [8] or by fluidizing them with highly adsorbing gases that increase the interparticle attractive force [9]. Application of external fields can stabilize fluidization too, as seen when an electric field is imposed on bubbling beds of semi-insulating Geldart B granules [10] and when a magnetic field is imposed on bubbling beds of ferromagnetic particles [11,12]. Generally, it is observed that the application of either electric or magnetic fields to these systems results in the formation of strings of polarized particles that eventually become jammed [10-12]. The fact that gas velocity and pressure drop can be related by means of a Carman equation shows that grains in a magnetically stabilized bed (MSB) are quiescent as held by interparticle forces [13]. The hydrodynamic, as well as heat and mass transfer, 
behavior of MSBs also indicates mechanical structuring of particles in strings $[14,15]$.

Most studies on magnetic field stabilization deal with the behavior of relatively large grains (Geldart B granular materials), showing just bubbling fluidization in the absence of field. Zhu and $\mathrm{Li}$ [16] have studied the behavior of Geldart's group $\mathrm{C}$ magnetic powders and the experiments demonstrated that channels and bubbles could be eliminated effectively by imposing a coaxial magnetic field. In our work we investigate the magnetofluidization behavior of naturally cohesive fine magnetite powder, which belongs to the class of Geldart A powders, i.e., the fluidized state of this powder can be naturally stabilized by van der Waals forces alone. Our aim is to study the effect of the field on stabilization and on the mechanical strength of the stably fluidized bed.

\section{A. Previous studies on magnetic stabilization}

Early empirical research on MSBs, dating back to the 1960s, was performed in East Europe as reviewed by Siegell [17]. Except for a few exceptions, the interest in magnetofluidized beds among western researchers was not ignited until the seminal paper published in 1979 by Rosensweig [18]. Rosensweig developed a linear stability analysis [19] for magnetically soft grains that was able to predict that the state of uniform fluidization could be stabilized by a coflow magnetic field against the growth of perturbations in voidage, bearing resemblance to the linear stability theory of fluidized beds stabilized by the natural van der Waals forces $[3,4]$. He further analyzed the rheology of MSBs. Visually, the MSB was free of agitation or solid recirculation, yet it could discharge through an orifice for magnetization fields below a threshold value. In the close vicinity of the transition between the stable and bubbling regimes, the transfer of the stabilized bed between processing vessels displayed liquidlike features [20]. Moreover, objects were readily immersed in the bed as in a liquid and a ping-pong ball that was initially rotated continued to spin for several seconds, indicating the low frictional resistance associated with buoyancy [18]. Further experimental studies have shown that the fluidity of MSBs continuously decreases as the magnetic field strength is increased. Invasive drawing-plate experiments were designed to have a quantitative measure of the yield stress of MSBs [21,22]. Lee [21] observed that appreciable yield stress appeared first at marginal stability and increased monotonically with further increase of the field strength. Siegell distinguished a transition from the stable to a frozen regime at high-intensity fields by measuring the ability of the fluidized bed to support high-density objects on its surface [23]. In the stabilized bed, objects placed on the bed surface, which had a higher bulk density than the fluidized bed, tended to sink, indicating a rather small yield stress. In the frozen bed, higher-density objects stood on the surface, indicating a high yield stress. Tilted bed experiments were also performed to differentiate between the stabilized and frozen regimes. When a vessel containing a stabilized bed was tilted, the top surface of the bed remained horizontal as if it were a liquid [23]. In contrast, when a vessel containing a frozen bed was tilted, the top surface remained normal to the vessel vertical axis [23]. An extended review of magnetofluidized bed studies can be found in Rosensweig's book [24].

Magnetofluidized beds of coarse Geldart B magnetite grains (of size above $100 \mu \mathrm{m}$ ) have been studied by Lee [22] and Hristov [25]. Lee [22] found that fluidized beds of coarse magnetite particles could be stabilized for coflow applied fields of the order of $H \simeq 1 \mathrm{kA} / \mathrm{m}$. Solid-discharge tests indicated that the stabilized bed fluidity was similar to the bubbling bed fluidity for fields of strength below a critical value. For example, at a gas velocity twice the minimum fluidization velocity, the bed was stabilized by a field of strength $H \simeq 6 \mathrm{kA} / \mathrm{m}$ and showed liquidlike behavior up to a field of strength $H \simeq 10 \mathrm{kA} / \mathrm{m}$. For field strengths above this so-called jelling field, the MSB fluidity sharply decreased with increasing field intensity. At a field intensity of approximately $18 \mathrm{kA} / \mathrm{m}$, the bed was frozen and the solid-discharge rate dropped to zero. Reports of frozen bed behavior for coflow magnetic fields of similar strength can be seen also in Ref. [25].

\section{EXPERIMENTAL SETUP AND MATERIAL}

The Sevilla powder tester (SPT) is a fluidized bed apparatus that enables us to measure the average particle volume fraction $\phi$ and tensile yield stress $\sigma_{t}$ of fluidized cohesive powders as a function of the gas velocity. The SPT functioning has been reported elsewhere in detail [26] and we give here only a brief summary. The powder sample is held in a vertically oriented cylindrical vessel $(2.54 \mathrm{~cm}$ internal diameter in the experiments reported in this paper) and rests on a porous plate that acts as gas distributor ( $5 \mu \mathrm{m}$ pore size). By means of a series of computer-controlled valves and a mass flow controller, a controlled flow of filtered and dried air is pumped through the powder bed while the gas pressure drop across it is read from a differential pressure transducer. The height of the bed, which gives an average value of the particle volume fraction $\phi$, is measured by means of an ultrasonic sensor placed on top of the vessel. This device can determine distance, with an accuracy smaller than local fluctuations in bed height, by sending an ultrasonic wave and measuring the time of reflection from the target.

The material used in the experiments is magnetite powder. The average particle diameter and particle density are $35 \mu \mathrm{m}$ and $5060 \mathrm{~kg} / \mathrm{m}^{3}$, respectively. This magnetic powder is tested as affected by a cross-flow externally imposed uniform magnetic field. The magnetic field strength is varied by adjusting the electrical current through a pair of square Helmholtz coils $\left(50 \times 50 \mathrm{~cm}^{2}\right)$ with each coil consisting of 500 turns of 2-mm-diameter. copper wire. The magnetic field strength is measured by a Hirst Magnetics gaussmeter using an axial probe with an accuracy less than $0.1 \mathrm{mT}$. Experimental measurements show that, within this experimental accuracy, the external field strength is homogeneous in the cell volume.

\section{MAGNETIC RHEOLOGY OF THE POWDER}

In order to find a physical interpretation for the data experimentally obtained it is important to magnetically charac- 


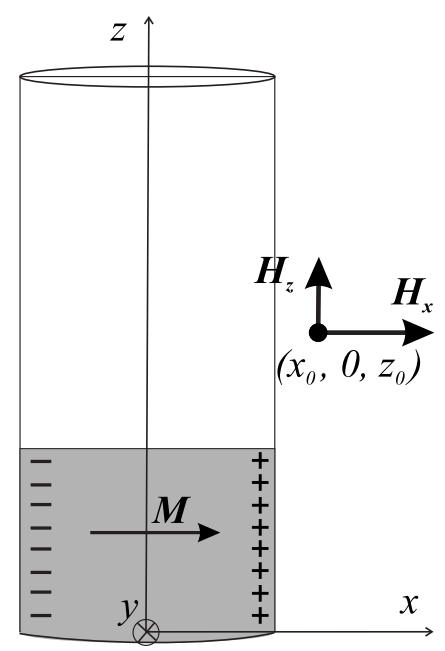

FIG. 1. Sketch of the 2.54-cm-diameter powder bed magnetized by an external field applied in the $x$ direction. The external field magnetizes the powder with a magnetization $\mathbf{M}$ in the $x$ direction. The $x$ component of the total magnetic field $H_{x}$ is measured by the probe at the point $x_{0}=2.51 \mathrm{~cm}, y_{0}=0, z_{0}=2.4 \mathrm{~cm}$. The settled bed height is $h=1.9 \mathrm{~cm}$.

terize the material used. Permanently magnetized magnetite (lodestone) is a magnetically hard material, with a saturation magnetization of around $500 \mathrm{kA} / \mathrm{m}[11,25,27,28]$ and a large coercive field, typically about $H_{c} \simeq 10 \mathrm{kA} / \mathrm{m}$ [27-30]. However, the magnetite powder employed in our experiments was artificially made by Xerox Co. and was not permanently magnetized. The size of magnetic domains in magnetite particles artificially manufactured by different routes was found to be of the order of $100 \mathrm{~nm}$ [31]. In order to induce permanent magnetization on artificial magnetite of comparable strength to that of permanently magnetized magnetite, fields strengths of $100 \mathrm{kA} / \mathrm{m}$ or higher are necessary [32]. In our study the applied field strength $H_{0}$ is typically below $5 \mathrm{kA} / \mathrm{m}$. For these small strength fields, the magnetic response of the material should be linear and reversible. The particle magnetization $\mathbf{M}_{p}$ would be therefore related to the externally imposed field $\mathbf{H}_{0}$ by means of the linear relationship $\mathbf{M}_{p}=\chi_{p} \mathbf{H}_{0}$, where $\chi_{p}$ is the initial particle susceptibility. Mills reported values of the initial susceptibility of natural magnetite about 2.3 for low fields, and always remaining $\$ 10$ [32]. Hunt et al. [33] reviewed plenty of data in the literature for magnetite rocks, which ranged from 1 to 5.7. Hunt et al. [33] also reviewed data on the initial susceptibility of magnetite particles of size between 0.01 and $100 \mu \mathrm{m}$, made from either crushing or crystal growing. The values reported of $\chi_{p}$ ranged between 2.5 and 10 .

We have obtained $\chi_{p}$ by measuring in our experimental setup the strength of the field at a fixed point (see Fig. 1) with and without the powder bed present. The powder bed is allowed to settle in the cylindrical vessel after being fluidized at a gas velocity $v_{g}=2 \mathrm{~cm} / \mathrm{s}$ to ensure reproducibility of the packing conditions. For a given current intensity $I$, the field measured $\mathbf{H}$ consists of the superposition of the external applied field $\mathbf{H}_{0}$, which is directed along the $x$ axis and was previously measured in the absence of the powder bed for the same current intensity, and the field $\mathbf{H}_{m}$ caused by the mag-

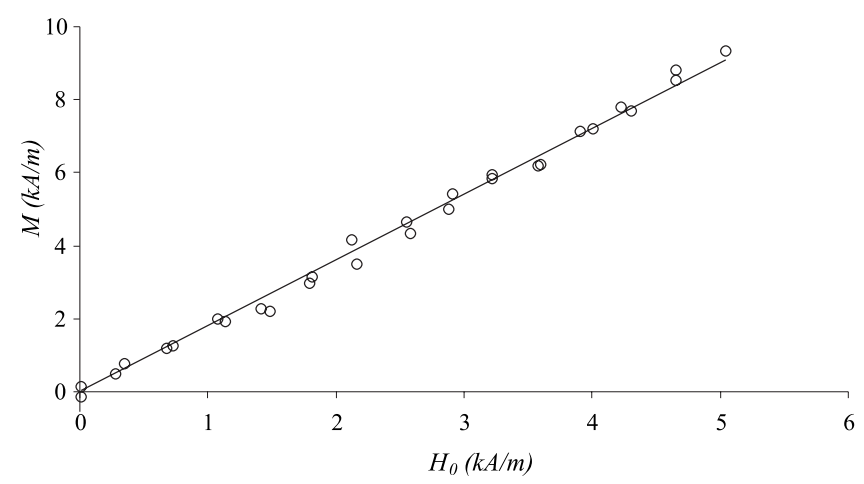

FIG. 2. Bulk magnetization of the magnetite powder bed used in the experiments as a function of the strength of the external field applied. The line is a linear fit to the data through the origin $M$ $=1.81 H_{0}$.

netized powder bed of height $h=1.9 \mathrm{~cm}$. Upon application of the external field the powder is assumed to be uniformly magnetized with a magnetization $\mathbf{M}=M \mathbf{u}_{x}$. The strength of the $x$ component of the field $\mathbf{H}_{m}$ is calculated numerically as $H_{m x}=0.039 \mathrm{M}$ at the point of measurement, which is located at a height $z_{0}=2.4 \mathrm{~cm}$ above the bottom of the bed and at a distance $x_{0}=2.51 \mathrm{~cm}$ from its vertical central axis (see Fig. 1). In Fig. 2 we show data on the bulk magnetization $M$ $=\left(H_{x}-H_{0}\right) / 0.039$ obtained from this procedure. It can be seen that the bulk magnetization increases linearly as the strength of the external field $H_{0}$ is increased, and can be well fitted by the equation $M=1.81 H_{0} \mathrm{~A} / \mathrm{m}$.

According to effective medium theories and numerical analysis on random granular materials [34], the bulk susceptibility $\chi$ of a bed of magnetically linear spherical particles of susceptibility $\chi_{p}$ located in a homogeneous environment of susceptibility $\chi_{0}$ can be calculated from the Bruggeman mixing rule

$$
(1-\phi) \frac{\chi_{0}-\chi}{3+\chi_{0}+2 \chi}+\phi \frac{\chi_{p}-\chi}{3+\chi_{p}+2 \chi}=0,
$$

where, in our case, the particle volume fraction is $\phi=0.48$, $\chi=1.81$, and $\chi_{0}=0$. From Eq. (1) we obtain a particle initial susceptibility $\chi_{p}=5.33$, which is comparable to the values reported in the literature for magnetite solids that were not permanently magnetized $[32,33]$. From this result, the relative deviation between the field measured by the probe and the external field can be obtained as $\Delta H / H_{0} \simeq 0.17$. In the fluidized bed case, the bulk magnetization $M$ is expected to be smaller since, on one hand, the agitation by the gas flow contributes to disorient the particle dipoles and, on the other, the particle volume fraction is decreased.

\section{EXPERIMENTAL RESULTS AND DISCUSSION}

\section{A. Stabilization by natural van der Waals forces}

Figure 3 shows data of the gas pressure drop across the magnetite powder bed $\Delta p$ measured as a function of the superficial gas velocity $v_{g}$ and in the absence of externally applied magnetic field ( $H$ off). In these tests, the powder is first driven to the bubbling regime by imposing a gas veloc- 


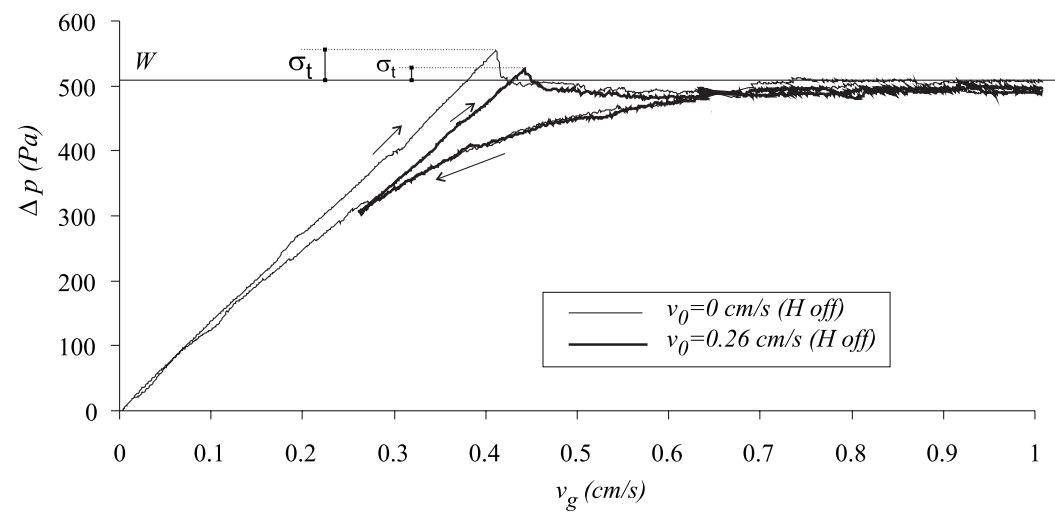

FIG. 3. Gas pressure drop across the magnetite powder bed as a function of superficial gas velocity during the fluidization-defluidization cycles in the absence of externally imposed magnetic field ( $H$ off). Examples of two cycles are plotted corresponding to an initial velocity $v_{0}$ $=0 \mathrm{~cm} / \mathrm{s}$ (bed settled under its own weight) and $v_{0}=0.26 \mathrm{~cm} / \mathrm{s}$ (bed settled under a remanent gas velocity $v_{0}=0.26 \mathrm{~cm} / \mathrm{s}$ ). The tensile strength $\sigma_{t}$ measurement for both states is indicated as well as the powder weight per unit area $W \simeq 510 \mathrm{~Pa}$.

ity $v_{g}=2 \mathrm{~cm} / \mathrm{s}$. Once the bubbling bed has reached a stationary state, in which it has lost the memory of its previous history $[35,36]$, the gas flow is suddenly turned off $\left(v_{g}=v_{0}\right.$ $=0 \mathrm{~cm} / \mathrm{s}$ ) and the bed is allowed to settle. The consolidation stress $\sigma_{c}$ in this initial state at the bottom of the sample is given by the powder's own weight per unit area, $W$, which is $W \simeq 510 \mathrm{~Pa}$. (Since we restrict our study to shallow beds, with heights smaller than the bed diameter, wall effects are negligible [37].) The settled powder layer is then subjected to a slowly increasing gas velocity. At first the bed structure is unperturbed and $\Delta p$ increases linearly as $v_{g}$ is increased (see Fig. 3). This linear behavior corresponds to Carman's law [38] for the resistance of porous solids to the passage of gas flow. The larger the porosity, or, equivalently, the smaller the particle volume fraction, the smaller the slope. At the point of minimum fluidization velocity $\left(v_{g}=v_{\mathrm{mf}} \simeq 0.39 \mathrm{~cm} / \mathrm{s}\right.$, see Fig. 3) $\Delta p$ balances $W$. At this point a powder with zero cohesion would become fluidized, yet the pressure drop across our naturally cohesive powder continues to increase above the minimum fluidization velocity. Above this point the gas flow puts the bed under tension, and as the tension builds up there comes a point at which the powder breaks in tension and the pressure drop falls to around the weight per unit area, $W$ (see Fig. 3). This is the point of incipient fluidization $\left(v_{g}=v_{\text {if }} \simeq 0.41 \mathrm{~cm} / \mathrm{s}\right)$. The condition for tensile yield is met first at the bottom of the bed, where the fracture of the bed is observed to start, as is theoretically expected [39]. Provided that wall effects are negligible, the tensile strength $\sigma_{t}$ of the settled powder is given by the difference between the pressure drop across the bed just before the breaking and the weight per unit area, $\sigma_{t}=(\Delta p)_{\max }-W$ $\simeq 43 \mathrm{~Pa}$. Further increase of the gas velocity gives rise to a state of heterogeneous fluidization, whose main characteristic is the propagation of the fracture in the upward direction while $\Delta p$ fluctuates around the powder weight per unit area, $W$. Large visible bubbles are seen to develop at $v_{g}=v_{b}$ $\simeq 0.8 \mathrm{~cm} / \mathrm{s}$, coinciding with a maximum of bed expansion.

If the gas velocity is now decreased from the bubbling regime, the typical hysteretic behavior of Geldart A powders becomes apparent at a gas velocity $v_{g}=v_{c} \simeq 0.65 \mathrm{~cm} / \mathrm{s}$, in which the powder bed is jammed, and $\Delta P<W$, indicating that part of the weight is sustained by the enduring interparticle contacts. As a consequence, there appears a consolidation stress $\sigma_{c}$ at the bottom of the bed, given by $\sigma_{c}=W$ $-\Delta p_{0}$, where $\Delta p_{0}$ is the gas pressure drop across the stably fluidized bed for a gas velocity $v_{0}<v_{c}$. If the gas flow during the defluidization part of the cycle is increased again from a value $v_{0}>0 \mathrm{~cm} / \mathrm{s}$, it is seen that $\Delta p$ increases again linearly as $v_{g}$ is increased, in agreement with Carman's law (see Fig. 3 , where we show an example curve for $v_{0}=0.26 \mathrm{~cm} / \mathrm{s}$ ). Note that the slope of this straight line is now smaller than the slope for the bed settled under its own weight $\left(v_{0}\right.$ $=0 \mathrm{~cm} / \mathrm{s}$ ), indicating a smaller particle volume fraction of the stably fluidized bed at a gas velocity $v_{0}$ as should correspond to an expanded state. The new pressure overshoot enables us to measure the tensile strength of the bed $\sigma_{t}$ in the underconsolidated state, which, as can be seen in Fig. 3, is now smaller.

In Fig. 4 the consolidation stress and tensile strength of the powder bed as measured through this process are shown as a function of $v_{0}$. As expected, $\sigma_{c}$ and $\sigma_{t}$ decrease as $v_{0}$ is increased. Within the accuracy of our pressure drop measurements, the tensile strength $\sigma_{t}$ becomes insignificant when the gas velocity $v_{0}$ surpasses the incipient fluidization velocity $v_{\text {if }} \simeq 0.41 \mathrm{~cm} / \mathrm{s}$. Thus, the measured yield stress of the fluid-
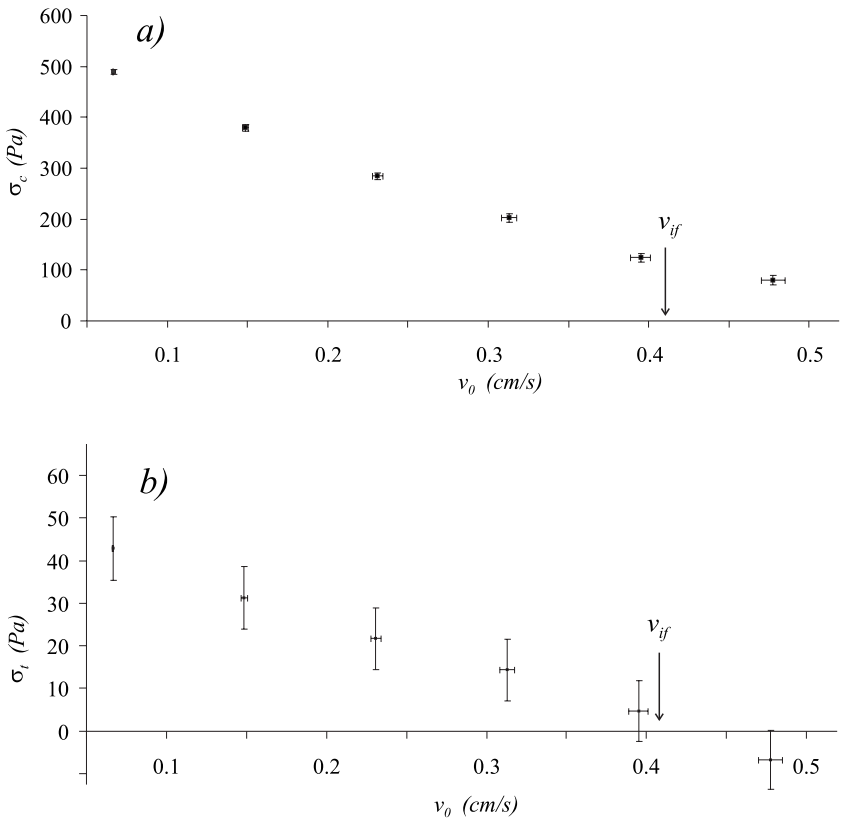

FIG. 4. (a) Consolidation stress of the powder bed as a function of the gas velocity during defluidization. (b) Tensile strength of the bed as a function of the remanent gas velocity during defluidization. The gas velocity at incipient fluidization $v_{\text {if }}$ of the bed settled under its own weight $\left(v_{\mathrm{if}}=0.41 \mathrm{~cm} / \mathrm{s}\right)$ is indicated. 
ized bed just stabilized by natural cohesive forces is practically negligible, albeit the bed possesses a solid structure as indicated by the nonvanishing value of the consolidation stress $\sigma_{c}$.

\section{Estimation of the tensile strength of the naturally stabilized bed}

The practically zero value of the tensile strength measured for gas velocities above incipient fluidization $\left(v_{0}\right.$ $>0.41 \mathrm{~cm} / \mathrm{s}$ ) may be rationalized from a simple estimation of the value of the interparticle attractive force $f$. In the absence of external magnetic field there will not be particle magnetization. Thus, the main interparticle attractive force is just the natural van der Waals attractive force [40], which is produced by the interaction of fluctuating molecular electric dipole fields. Assuming that retardation effects are negligible and that the interaction between molecules is pairwise, Hamaker [41] summed up all the interactions between two spherical and rigid particles at contact with diameters $d_{1}$ and $d_{2}$ and arrived at the approximate expression for the attractive force

$$
f_{\mathrm{vdW}} \simeq \frac{A d^{*}}{12 z_{0}^{2}},
$$

where $A$ is the Hamaker constant, $d^{*}=d_{1} d_{2} /\left(d_{1}+d_{2}\right)$ is the reduced diameter, and $z_{0} \simeq 3-4 \AA$ is the distance of closest approach between two molecules. Because of the short range of the molecular interaction, the van der Waals force is actually determined by the local radius of curvature of the surface asperities at contact. Therefore the typical size of the surface asperities $d_{a}$ must be used in Eq. (2) instead of the particle diameters. A typical value reported for the size of surface asperities of fine powder particles is $d_{a} \simeq 0.2 \mu \mathrm{m}$ [3], while the reported values of $A$ for oxides ranges between $1.06 \times 10^{-19}$ and $1.55 \times 10^{-19} \mathrm{~J}[42,43]$. Thus we can estimate an attractive force $f \simeq f_{\mathrm{vdW}} \simeq 10 \mathrm{nN}$ between our experimental particles.

The bulk tensile strength of the powder that arises from the existence of an interparticle attractive force $f$ can be estimated by means of the Rumpf averaging equation $[44,45]$ as

$$
\sigma_{t} \sim f \frac{\zeta \phi}{\pi d_{p}^{2}},
$$

where $\zeta$ is the coordination number (average number of contacts per particle), which can be related to the particle volume fraction $\phi$ by the equation $\zeta \simeq(\pi / 2)(1-\phi)^{-3 / 2}$ (Ref. [46]). Using as typical values $f=f_{\mathrm{vdW}}=10 \mathrm{nN}, d_{p}=35 \mu \mathrm{m}$, and $\phi=0.4$, it is estimated that $\sigma_{t} \simeq 4 \mathrm{~Pa}$, which is about our experimental indeterminacy. It is explainable therefore that the measured tensile strength of the fluidized bed, stabilized by the attractive van der Waals forces alone, is negligible. Note that for coarse Geldart B particles $\left(d_{p}>100 \mu \mathrm{m}\right)$ the tensile strength calculated would be even smaller, which explains the nonexistence, in the absence of wall restraining effects, of a pressure overshoot in the pressure drop cycles usually shown in the literature for coarse granules.
As the gas velocity is decreased below the incipient fluidization velocity $\left(v_{0}<0.41 \mathrm{~cm} / \mathrm{s}\right)$, the powder is increasingly consolidated due to its own weight force and plastic deformation of interparticle contacts might possibly increase the interparticle attractive force [47], thus increasing the tensile strength. The tensile strength of a $h \simeq 1 \mathrm{~cm}$ depth layer $\left(\sigma_{c} \simeq \rho_{p} \phi g h \simeq 200 \mathrm{~Pa}\right)$ of the magnetite powder used in the present study has been reported elsewhere as measured using a centrifuge tester [48]. The measured value was $\sigma_{t} \simeq 15 \mathrm{~Pa}$, which is close to the result obtained in the present study for a similar consolidation stress $\sigma_{c}$ reached when the bed is settled under a remanent gas velocity $v_{0} \simeq 0.26 \mathrm{~cm} / \mathrm{s}$ (see Fig. 4). Equation (2) cannot explain, however, the enhancement of interparticle attractive force due to plastic deformation of interparticle contacts due to consolidation stresses. In order to take into account this effect, more elaborated theories, such as the Mesarovic and Johnson theory, are needed (see [40] for a detailed review). Since we are concerned in this paper just with the effect of the magnetic field, we will focus our attention on the tensile strength measured for fluidized states of small consolidation, where plastic deformation does not have a relevant effect.

\section{B. Stabilization in the presence of a magnetic field}

In order to test the effect of a cross-flow magnetic field on the fluidization behavior, the measuring process described above has been performed for different values of the applied magnetic field strength and following different procedures. We first looked at the effect of the magnetic field on the transition to the stable state. This is accomplished by applying the field when the bed is in the initial bubbling regime. Then, the gas velocity is slowly decreased while the field strength is kept constant. In this way, the gas velocity at the transition to the stable state $v_{c}$ in the presence of the external magnetic field can be identified at the point at which the bed is jammed and $\Delta p$ becomes smaller than $W$. Since the gas flow and the magnetic field are two independent variables, an alternative way to stabilize the bed is to fix the gas velocity above the bubbling velocity in the absence of magnetic field and then apply the magnetic field, slowly increasing its strength until bubbles are suppressed and the bed is jammed.

The data for the transition velocity $v_{c}$ from the bubbling regime to the stable state are plotted in Fig. 5 as a function of the magnetic field strength (left axis). A remarkable result is that, in the presence of magnetic field, the jamming transition occurs at higher gas velocities as compared with the transition velocity in the absence of external field. The field effect becomes noticeable for strengths roughly above $2 \mathrm{kA} / \mathrm{m}$. For $H \gtrsim 2 \mathrm{kA} / \mathrm{m}, v_{c}$ increases steadily as the magnetic field strength is increased. It is also seen that, within the experimental scatter, the data fit the same trend independently of which variable, either $H$ or $v_{g}$, was fixed in the experimental procedure.

Figure 6 shows data on the measured values of the particle volume fraction of the bed at the transition to the stable state $\left[\phi\left(v_{c}\right)=\phi_{c}\right]$ as a function of the magnetic field strength. Generally, magnetic stabilization gives rise to more expanded structures (smaller $\phi_{c}$ ), which indicates that the mag- 


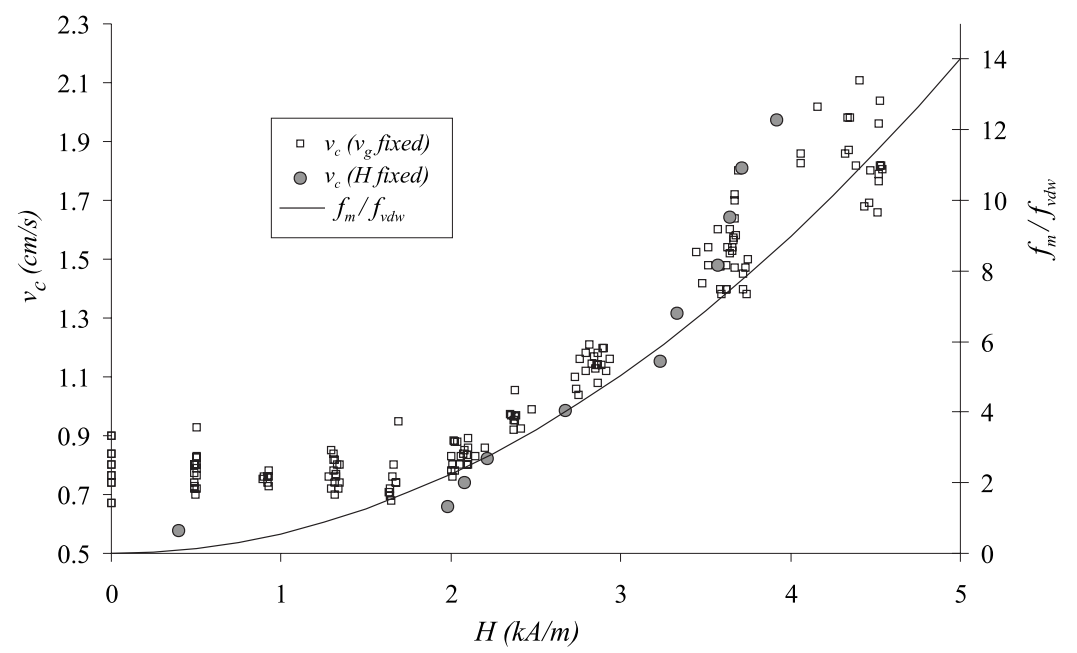

FIG. 5. Left axis: Gas velocity at the transition to the stable fluidization regime $v_{c}$ as a function of the magnetic field strength according to the two procedures employed $\left(v_{g}\right.$ fixed: the gas velocity is fixed at bubbling and the magnetic field strength is slowly increased from zero; $H$ fixed: the magnetic field strength is fixed and the gas velocity is slowly decreased from bubbling). Right axis: ratio of the calculated attractive interparticle magnetic force to the attractive interparticle van der Waals force. netically induced attractive forces have an effect on the packing arrangement of the particles in the stabilized bed. In agreement with the previous analysis on $v_{c}$, it is seen that the effect of the field becomes relevant for field strengths above $2 \mathrm{kA} / \mathrm{m}$. Note, however, that the values of $\phi_{c}$ are smaller in the fixed gas velocity experiments. This effect could be due to the quasistatic increase of the field strength at fixed gas velocity, which would allow for further particle restructuring at jamming.

The extension of the stable fluidization interval to higher gas velocities in the presence of the magnetic field can be attributed to the increase of the interparticle attractive force due to the magnetization of the particles. A rigorous calculation of the force between magnetized particles would require a large number of multipolar moments when the particles are in contact [49]. Nevertheless, in the range of field strengths applied, the dipolar approximation can be used for an estimation of order of magnitude of the contact force [49]. The attractive force between two aligned dipoles of moment $m_{p}$ separated by a distance $d_{p}$ is given by [50]

$$
f_{m}=\frac{3 \mu_{0} m_{p}^{2}}{2 \pi d_{p}^{4}},
$$

where $\mu_{0}$ is the permeability of free space $\left(4 \pi \times 10^{-7} \mathrm{H} / \mathrm{m}\right)$. According to our rheological measurements, in the presence of an external field, the magnetite particles would be magnetized with a dipolar moment $m_{p}=\chi_{p} H_{0}(1 / 6) \pi d_{p}^{3}$, where $\chi_{p}$ $=5.33$. In Fig. 5 we have plotted the ratio $f_{m} / f_{\text {vdw }}$ (right axis) as a function of the magnetic field strength. It is observed that the effect of the magnetic field becomes noticeable at the point in which the magnetic force becomes around twice the van der Waals force. For smaller fields, the magnetic force is comparable or smaller than the van der Waals force and there is not an observable effect of the magnetic field on the jamming transition. As the magnetic field is increased above $H$ $\simeq 2 \mathrm{kA} / \mathrm{m}, v_{c}$ increases proportionally to the increase of $f_{m} / f_{\mathrm{vdW}}\left(\Delta v_{g} \propto f_{m} / f_{\mathrm{vdW}} \propto H_{0}^{2}\right)$. Thus, the data suggest that the transition to the stable state in the presence of the magnetic field is ruled by the strength of the dipole-dipole attractive force.

\section{Tensile strength of the magnetically stabilized bed}

We have investigated whether the tensile strength $\sigma_{t}$ of the stably fluidized bed is affected by the external magnetic field. The procedure followed to measure the tensile strength is the same as previously described in the absence of the magnetic field ( $H$ off; see Fig. 3). In our experiments the application of the field has been performed in two different operation modes. In one mode (hereafter named $H$ on-on) the

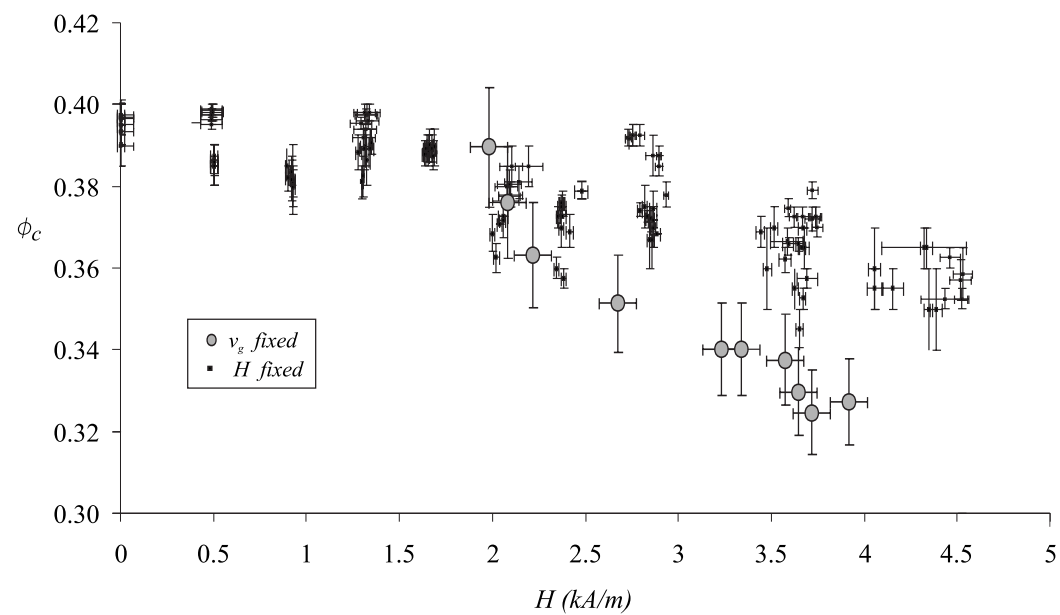

FIG. 6. Particle volume fraction $\phi$ of the fluidized bed at magnetic stabilization vs the strength of the magnetic field according to the two procedures employed $\left(v_{g}\right.$ fixed: the gas velocity is fixed at bubbling and the magnetic field strength is slowly increased from zero; $H$ fixed: the magnetic field strength is fixed and the gas velocity is slowly decreased from bubbling). 


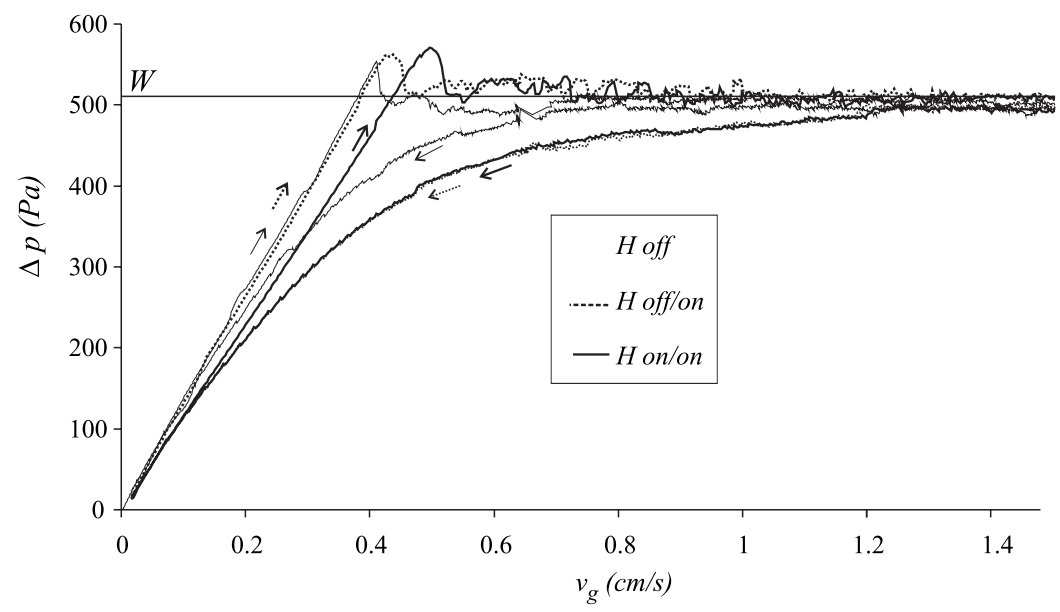

FIG. 7. Fluidization-defluidization cycles according to both modes of operation for $v_{0}$ $=0 \mathrm{~cm} / \mathrm{s}$ for a field strength $H=3 \mathrm{kA} / \mathrm{m}$. Fluidization-defluidization cycle in the absence of field ( $H$ off) is shown for comparison.

bed is driven to bubbling by applying a high gas velocity $\left(v_{g}=2 \mathrm{~cm} / \mathrm{s}\right)$ and the magnetic field is turned on. Then, the bed is allowed to settle at a remanent gas velocity $v_{0}$ while the magnetic field strength is kept constant. In this state the bed is stabilized by both natural and magnetically induced interparticle cohesive forces. In the other operation mode (hereafter named $H$ off-on), the bed is driven to bubbling and allowed to settle at a gas velocity $v_{0}$ in the absence of magnetic field. In this state the bed remains stabilized just by the natural interparticle cohesive forces. After the bed is naturally stabilized, the magnetic field is turned on and the tensile strength in the presence of the field is measured.

Fluidization-defluidization cycles for the bed initially settled under its own weight $\left(v_{0}=0 \mathrm{~cm} / \mathrm{s}\right)$ and for both modes of operation are plotted in Fig. 7. Data measured in the absence of external magnetic field ( $H$ off) are also shown for comparison. It is seen that, as the gas velocity is increased from zero, the gas pressure drop in the $H$ off-on operation mode increases at the same rate as it does in the absence of field, which indicates that application of the field after the bed is settled does not change the bed packing structure. Note, however, that the pressure overshoot and the incipient fluidization velocity is slightly increased, which indicates that the tensile strength of the bed is slightly increased by application of the field. On the other hand, the rate of increase of the gas pressure drop in the $H$ on-on operation mode is relatively decreased. This is a consequence of the higher porosity of the bed that was fluidized and settled in the presence of the field ( $H$ on-on). Remarkably, the tensile strength and incipient fluidization velocity are appreciably increased in this operation mode. As seen previously, Fig. 7 shows that the transition velocity $v_{c}$ to the stable state, which is identified by the beginning of the hysteretic behavior as the gas velocity is decreased, is delayed in the presence of the field. Note also that the pressure drop curves for decreasing gas velocities are the same in both modes of operation, which indicates that the bed has lost memory of its initial state when it bubbles at high velocities in the presence of the field.

Figure 8 shows tensile strength data for the bed initially settled at gas velocities similar to incipient fluidization and above. It is observed that the bed is provided with an appreciable tensile strength only for field strengths above $H$ $\simeq 2 \mathrm{kA} / \mathrm{m}$. Moreover, it can be noticed that the bed is provided with a larger tensile strength when it is operated in the $H$ on-on mode.

\section{Theoretical estimation of the tensile strength of the magnetically stabilized bed}

The tensile strength of a packed bed of magnetically linear grains subjected to a magnetic field was calculated in Ref. [51]. By applying the Maxwell stress tensor to a thin pill box enclosing a material surface, the surface normal stress in a structurally and magnetically isotropic, linearly magnetizable powder is obtained [51] as

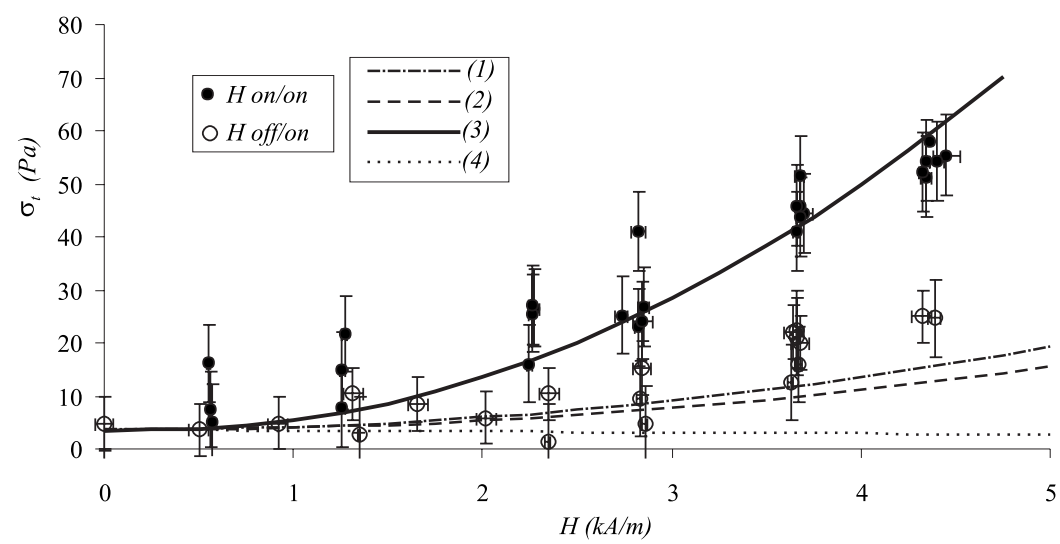

031306-7
FIG. 8. Tensile strength of the stabilized bed as a function of the strength of the magnetic field for gas velocities similar to incipient fluidization and larger according to both operation modes. The lines represent the predictions by the (1) Jones et al. discrete model [Eq. (8), $\alpha=0.16, \chi$ $=1.81$ ]; (2) Jones et al. continuum model [Eq. (8), $\alpha=0.125, \chi=1.81$ ]; (3) discrete model for a tetragonal lattice proposed in this paper (Sec. IV D 1); (4) Rumpf model including as interparticle force just the attractive van der Waals force [Eq. (3)]. 

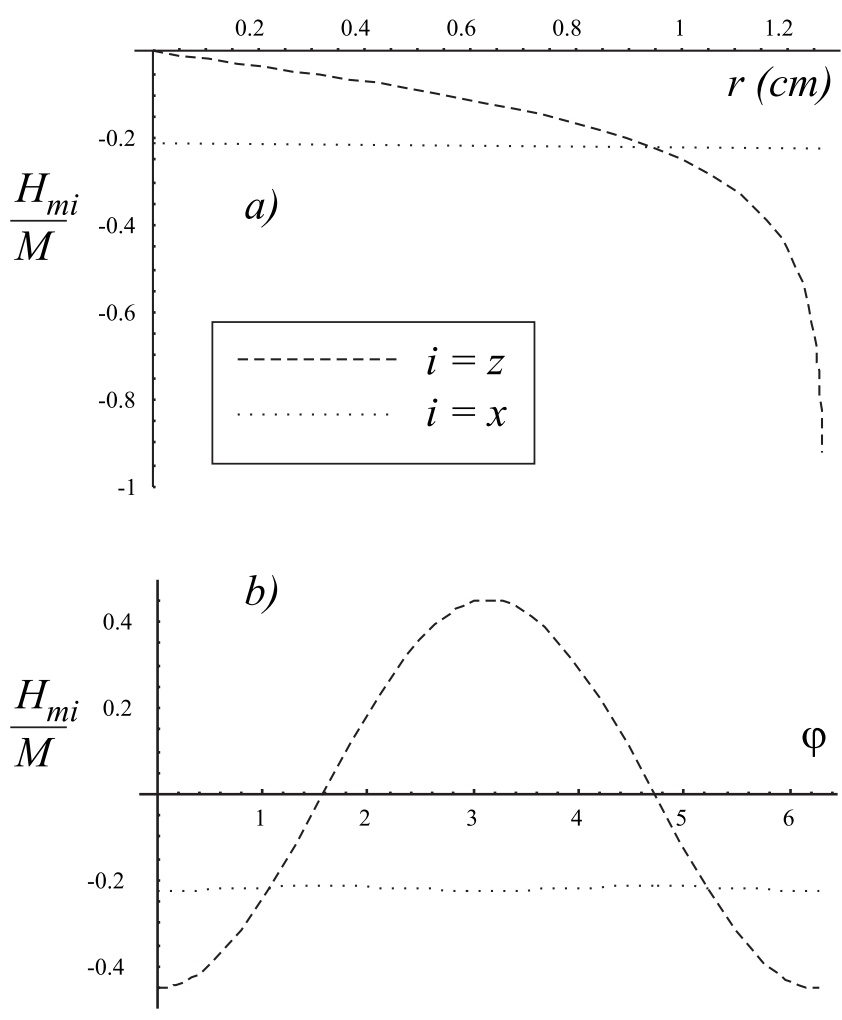

FIG. 9. Radial (a) and angular (b) distributions of the $x$ and $z$ components of the field created by the magnetized powder bed at the bottom plane $(z=0)$. In (a) $\varphi$ is fixed to $\varphi=0$. In (b) $r$ is fixed to $1.2 \mathrm{~cm}$.

$$
\sigma_{s}=\frac{\mu_{0}}{2} \chi^{2} H^{2}\left(\frac{2}{3} \cos ^{2} \theta-\frac{1}{3} \sin ^{2} \theta\right),
$$

where $H$ is the magnetic field strength within the powder, $\theta$ is the angle between the internal field and the surface normal, and $\chi$ is the bulk susceptibility of the powder. This equation clearly shows that the tensile strength is direction dependent (relative to the field), which gives the material anisotropic strength properties. Using this equation for the plane $z=0$ of our powder bed, which is the fracture surface in our tensile strength measurements, the surface stress is

$$
\sigma_{s}(r, \varphi)=\frac{\mu_{0}}{2} \chi^{2}\left(\frac{2}{3} H_{z}(r, \varphi, 0)^{2}-\frac{1}{3}\left[H_{x}(r, \varphi, 0)^{2}+H_{y}(r, \varphi, 0)^{2}\right]\right)
$$

where the polar coordinates $r$ and $\varphi$ have been used. Figure 9 shows the distributions of the $x$ and $z$ components of the magnetic field $\left(H_{m x}, H_{m z}\right)$ created by the magnetized powder bed, which are calculated by assuming a uniform magnetization $M$ (the $y$ component $H_{m y} \lesssim 0.005 M$ is negligible). It is seen that there exists an almost constant $x$ component of the field, $H_{m x} \simeq-0.215 M \simeq-0.5 H_{0}$, where we have used $M$ $=\chi H_{0}(\chi=1.81)$. Thus $H_{x}=H_{0}+H_{m x} \simeq 0.5 H_{0} . H_{m z}$ takes its maximum values on the $x$ and $y$ axes, along which it is zero at the origin and grows as $r$ approaches the cell size limit $(r=1.27 \mathrm{~cm})$. The average value of $H_{m z}^{2}$ is $\left\langle H_{m z}^{2}\right\rangle \simeq 0.22 M$ $\simeq 0.5 H_{0}$. According to these results the average surface stress at the bottom of the bed is $\left\langle\sigma_{s}\right\rangle=0.125 \mu_{0} \chi^{2} H_{0}^{2}$. Note that $\sigma_{s}$ is positive, which means that, due to the field produced by the own powder magnetization, there will be a cohesive stress at the plane $z=0$.

Jones et al. [51] derived an equation similar to Eq. (5) from the calculation of the interacting forces between particle dipoles aligned with the applied field and ordered in a cubic lattice. Using the dipole approximation, Jones et al. arrived at

$$
\sigma_{s}=\left(3 \mu_{0} / 4 \pi\right) \chi^{2} H^{2}\left(1.83 \cos ^{2} \theta-0.96 \sin ^{2} \theta\right) .
$$

Taking our averaged values of the $x$ and $z$ components of the field, it is $\left\langle\sigma_{s}\right\rangle=0.16 \mu_{0} \chi^{2} H_{0}^{2}$, which is similar to the result obtained from the continuum model of an isotropic material. It should be remarked, however, that the predicted cohesive stress will depend on the assumed packing. For example, an hexagonal packing gives a much smaller cohesion [52]. To our knowledge neither Eq. (5) nor Eq. (7) has been checked with experimental data yet. The lack of this experimental evidence is also recognized by Jones in his book [52].

According to these models the total tensile strength at $z=0$ would be given by

$$
\sigma_{t}=\alpha \mu_{0} \chi^{2} H_{0}^{2}+f_{\mathrm{vdw}} \frac{\zeta \phi}{\pi d_{p}^{2}}
$$

where $\alpha$ is 0.125 for the continuum approach and 0.16 for the discrete approach. In Eq. (8) the natural cohesive stress due to the interparticle van der Waals force is simply added to the magnetic surface stress.

In Fig. 8 we have plotted the tensile strength as calculated from the Jones et al. continuum and discrete models [Eq. (8)]. These theoretical calculations are compared with experimental data obtained for the magnetically stabilized bed at gas velocities $v_{0}$ similar to incipient fluidization and larger (at these gas velocities the tensile strength arising from consolidation due to sample weight, which is not considered in the theoretical equations, is not appreciable). It is seen that Eq. (8) fits well to the data measured by means of the $H$ off-on operation mode. On the other hand, the data measured by means of the $H$ on-on operation mode are clearly above the model predictions.

\section{Magnetic cohesion of particles in a tetragonal lattice}

Restructuring of the particles does not seem possible in the $H$ off-on operation mode since the field is applied after the bed is naturally stabilized and the particles have lost their freedom to rearrange. In this state it is thus unlikely that the field changes the packing structure of the isotropically jammed bed. In contrast, the packing of the stabilized bed in the $H$ on-on mode should be affected by the formation of structures of aligned particle dipoles due to particle magnetization and reorientation during bubbling and settling while the field is kept on. Particle chaining in the $H$ on-on mode might give rise to an anisotropic structure in the stabilized bed that should explain the enhancement of the cohesive strength seen experimentally.

The effect of anisotropy can be estimated by calculating the cohesive stress of a tetragonal crystal lattice of magnetic 


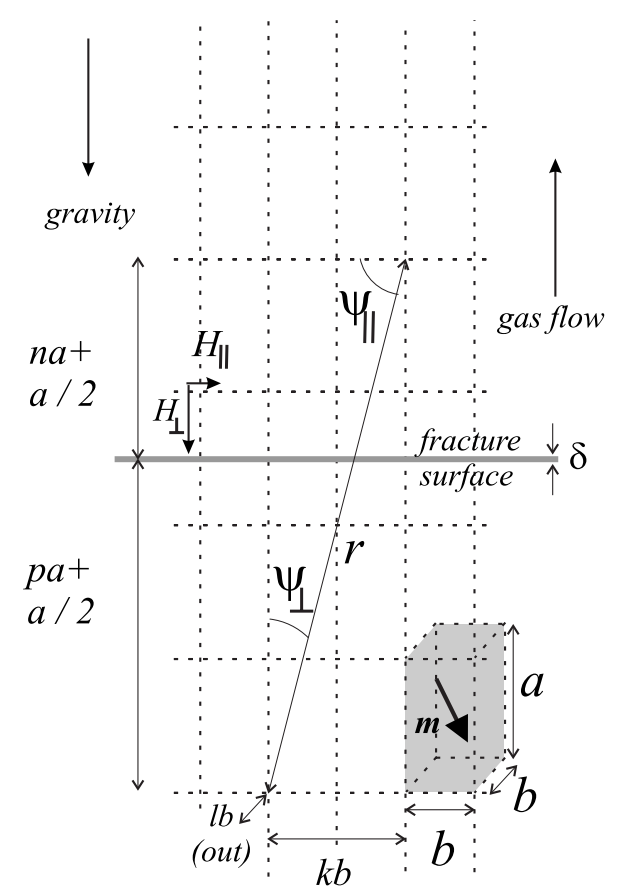

FIG. 10. Tetragonal lattice $(b \times b \times a)$ of magnetic dipoles oriented along the field lines for calculating the surface magnetic stress. $l b$ denotes the distance between dipoles in the perpendicular direction to the plane of the figure.

dipoles. In this section we extend the calculations by Jones et al. [51] on the cohesive stress of a cubic crystal lattice of magnetic dipoles to a tetragonal crystal lattice, which results from stretching the cubic lattice along one of its lattice vectors. Thus, the lattice unit becomes a rectangular prism with a horizontal square base $(b \times b)$ and a vertical height $a$ (see Fig. 10).

Let us consider a tetragonal lattice of dipoles having cell volume $(b \times b \times a)$ as shown in Fig. 10. The magnetic surface stress on an area $b \times b$ would be given by

$$
\sigma_{s}=\frac{1}{b^{2}}\left(\frac{\partial U}{\partial \delta}\right)_{\delta=0}
$$

where $U$ is the magnetic potential energy, which can be obtained by summing up the interaction energies between dipole pairs, $U_{12}$. The interaction energy $U_{12}$ between two parallel dipoles of equal magnetic moments $m=\chi H b^{2} a$ is given by [53]

$$
U_{12}=\frac{\mu_{0}}{4 \pi} \frac{m^{2}}{r^{3}}\left(1-3 \sin ^{2} \psi\right)
$$

where $r$ is the distance between the dipoles, and $\psi$ is the angle between the dipole moment direction and the direction of the magnetic field. According to Fig. 10 we would have $\psi=\psi_{\perp}$ for the component of the field perpendicular to the fracture surface $H_{\perp}$, and $\psi=\psi_{\|}=\pi / 2-\psi_{\perp}$ for the component of the field parallel to the fracture surface $H_{\|}$. Thus,

$$
\sigma_{s}=\frac{1}{b^{2}} \sum_{n=0}^{\infty} \sum_{p=0}^{\infty} \sum_{k=-\infty}^{\infty} \sum_{l=-\infty}^{\infty}\left(\frac{\partial U_{12}}{\partial \delta}\right)_{\delta=0},
$$

where the integers $n, p, k, l$ give the orientation of the dipoles relative to each other and the distance $r$ between them,

$$
\begin{gathered}
\sin ^{2} \psi_{\perp}=\frac{b^{2}\left(k^{2}+l^{2}\right)}{r^{2}}, \\
r^{2}=[a(n+p+1)+\delta]^{2}+b^{2}\left(k^{2}+l^{2}\right) .
\end{gathered}
$$

In the case of a cubic lattice $(b=a)$, the surface stress is given Eq. (7). For $b \neq a$, the surface stress depends on the ratio $b / a$. The case $b / a<1$ seems more appropriate to model an anisotropic structure consisting of dipole chains preferentially oriented in the direction of gas flow and gravity field. In Fig. 8 we have plotted the cohesive surface stress calculated for $b / a=0.44$, which is increased and becomes closer to the experimental data using the $H$ on-on operation mode. This result suggests that keeping the magnetic field on during liquidlike bubbling and settling ( $H$ on-on operation mode) enhances particle chaining, which modifies the arrangement structure of the particles in the stabilized state and thus introduces structural anisotropy. In contrast, this phenomenon cannot take place in the $H$ off-on operation mode since the magnetic field is applied after the particles are already jammed quasi-isotropically.

Our results would suggest that particle chaining is likely in the $H$ on-on operation mode and, according to the fit by the anisotropic structure model, chains would consist on average of just a few particles. This is in agreement with results inferred from experimental works in which MSB structures are studied. Rosensweig et al. [54] casted a MSB of steel spheres in a matrix of a polymerizing fluid. From these castings, Rosensweig et al. found that the bed porosity was constant to within $0.2 \%$ over the bed cross section, regardless of the bed expansion, which ranged up to $40 \%$. Moreover, the experiments indicated that at bed expansions of $16 \%, 30 \%$, and $37 \%$, particles remained randomly distributed, as in an unexpanded bed. However, for a $44.7 \%$ bed expansion, there was some degree of particle chaining. The chain length was very small, being less than $0.6 \mathrm{~cm}$, compared with the overall bed length of $11.6 \mathrm{~cm}$. Additionally, Rosensweig [54] measured the magnetic permeability of a MSB of steel spheres and the results were in good agreement with permeability models but indicated that a small degree of chaining developed, just on the order of 2-3 particles per chain.

\section{CONCLUSIONS}

In this work we have studied the behavior of a magnetofluidized bed of naturally cohesive $35-\mu \mathrm{m}$-diameter artificially made magnetite particles. Our rheological measurements show that the magnetic response of these particles to an externally imposed magnetic field is linear and can be characterized by a particle susceptibility $\chi_{p} \simeq 5.33$ in the range of field strengths applied.

In the absence of applied magnetic field, the fluidized bed of unmagnetized particles exhibits a typical Geldart A behav- 
ior, characterized by an interval of gas velocities in which the bed is stabilized by natural van der Waals forces. For gas velocities between incipient fluidization velocity $\left(v_{\text {if }}\right.$ $\simeq 0.41 \mathrm{~cm} / \mathrm{s}$ ) and $v_{c} \simeq 0.65 \mathrm{~cm} / \mathrm{s}$, the bed exhibits a stable fluidization behavior, which is characterized by a nonzero consolidation stress, i.e., the gas pressure drop is below the powder weight per unit area. In this jammed state, the measured tensile strength is almost negligible, which agrees with theoretical estimations of the tensile strength based on the Rumpf model and interparticle van der Waals forces.

Magnetofluidization experiments have been performed according to different procedures. A first procedure consists of driving the bed to bubbling fluidization in the presence of an applied magnetic field. Then the gas velocity is slowly decreased to look for the transition to stable fluidization. A second procedure consists of fixing the gas velocity in the bubbling regime while the magnetic field strength is slowly increased from zero. It has been seen that, independently of the procedure employed, the bubbling bed may be stabilized at gas velocities well above $v_{c} \simeq 0.65 \mathrm{~cm} / \mathrm{s}$ in the presence of the field. The minimum field strength necessary to extend the interval of stable fluidization and enhance bed expansion is about $2 \mathrm{kA} / \mathrm{m}$. At this field strength the interparticle magnetic force is estimated to be about twice the van der Waals force, which means that the magnetic interaction becomes relevant in the dynamics of the system. As the strength of the field is increased, the interval of stable fluidization increases proportionally to the increase of the ratio of interparticle magnetic force to van der Waals force. Maximum expansion of the magnetically stabilized bed is seen to be further enhanced when the bed is first driven to bubbling and the field strength is quasistatically increased from zero.

An additional study has consisted of measuring the tensile strength of the stably fluidized bed as affected by the external magnetic field. Application of a magnetic field to the naturally stabilized bed ( $H$ off-on operation mode) gives rise to a small increment of its tensile strength. On the other hand, application of the field during fluidization and settling of the fluidized bed ( $H$ on-on operation mode) produces an appreciable increment of the tensile strength. A reasonable explanation is that, in the fluidized state, particles are free to move and, being magnetized by the external field, are prone to form particle chains that strengthen the bed when the gas velocity is decreased ( $H$ on-on operation mode). In contrast, if the magnetic field is turned on once the bed is naturally stabilized ( $H$ off-on operation mode), the magnetized particles are constrained in fixed positions and their restructuring is not allowed. The experimental data on the tensile strength of the stabilized bed using the $H$ off-on operation mode adjusts to the surface stress predicted by a continuum model on an isotropic magnetically linear material. The enhancement of the tensile strength of the stabilized bed using the $H$ on-on operation mode can be explained by a discrete model using a tetragonal lattice of magnetic dipoles. As inferred from the previous study on the transition to the stable state, this suggests that in the $H$ on-on operation mode particle chains are formed in the bubbling state and settle preferentially oriented in the direction of the gas flow. Moreover, the increment of the tensile strength becomes appreciable for magnetic field strengths above $2 \mathrm{kA} / \mathrm{m}$, which is the minimum field strength necessary for magnetic extended stabilization and dominance of the magnetic dipole-dipole force over the van der Waals force.

\section{ACKNOWLEDGMENTS}

We acknowledge the Spanish Government Agency Ministerio de Ciencia y Tecnologia (Contract No. FIS2006-03645) and Junta de Andalucia (Contract No. FQM 421). We are grateful to Professor J. Y. Hristov, who helped us to build the magnetic system for field generation during his sabbatical stay in our laboratory and with whom we had numerous useful discussions.
[1] J. P. Constantineau, J. R. Grace, C. J. Lim, and G. G. Richards, Chem. Eng. Sci. 62, 70 (2007).

[2] D. Geldart, Powder Technol. 7, 285 (1973).

[3] K. Rietema, The Dynamics of Fine Powders (Elsevier, London, 1991).

[4] R. Jackson, The Dynamics of Fluidized Particles (Cambridge University Press, Cambridge, U.K., 2000).

[5] S. Sundaresan, Annu. Rev. Fluid Mech. 35, 63 (2003).

[6] J. M. Valverde, A. Castellanos, and M. A. S. Quintanilla, Phys. Rev. Lett. 86, 3020 (2001).

[7] J. M. Valverde, M. A. S. Quintanilla, A. Castellanos, and P. Mills, Phys. Rev. E 67, 016303 (2003).

[8] J. P. K. Seville and R. C. Clift, Powder Technol. 37, 117 (1984).

[9] H.-Y. Xie and D. Geldart, Powder Technol. 82, 269 (1995).

[10] T. W. Johnson and J. R. Melcher, Ind. Eng. Chem. Fundam. 14, 146 (1975).

[11] J. Y. Hristov, Powder Technol. 87, 59 (1996).

[12] M. J. Rhodes, X. S. Wang, A. J. Forsyth, K. S. Gan, and S.
Phadtajaphan, Chem. Eng. Sci. 56, 5429 (2001).

[13] J. Arnaldos, J. Casal, A. Lucas, and L. Puigjaner, Powder Technol. 44, 57 (1985).

[14] W. Resnick, Y. Zimmels, and D. Boadi, IEEE Trans. Magn. 24, 757 (1988).

[15] J. Casal and J. Arnaldos, Powder Technol. 64, 43 (1991).

[16] Q. Zhu and H. Li, Powder Technol. 86, 179 (1996).

[17] J. H. Siegell, Powder Technol. 57, 213 (1989).

[18] R. E. Rosensweig, Science 204, 57 (1979).

[19] R. E. Rosensweig, Ind. Eng. Chem. Fundam. 18, 260 (1979).

[20] J. H. Siegell and C. A. Coulaloglou, Powder Technol. 39, 215 (1984).

[21] W. K. Lee, AIChE Symp. Ser. 79, 87 (1983).

[22] W. K. Lee, Powder Technol. 64, 69 (1991).

[23] J. H. Siegell, Powder Technol. 55, 127 (1988).

[24] R. E. Rosensweig, Ferrohydrodynamics (Dover, New York, 1997).

[25] J. Y. Hristov, Powder Technol. 97, 35 (1998).

[26] J. M. Valverde, A. Castellanos, A. Ramos, A. T. Perez, M. A. 
Morgan, and P. K. Watson, Rev. Sci. Instrum. 71, 2791 (2000).

[27] L. G. Parry, Philos. Mag. 11, 303 (1965).

[28] X. Lu and H. Li, Powder Technol. 107, 66 (2000).

[29] Y. A. Liu, R. Keith Hamby, and R. D. Colberg, Powder Technol. 64, 3 (1991)

[30] D. Jiles, Magnetism and Magnetic Materials (Chapman \& Hall, London, 1996).

[31] G. F. Goya, T. S. Berquo, F. C. Fonseca, and M. P. Morales, J. Appl. Phys. 94, 3520 (2003).

[32] A. A. Mills, Ann. Sci. 61, 273 (2004).

[33] C. P. Hunt, B. M. Moskowitz, and S. K. Banerjee, in Rocks Physics and Phase Relations: A Handbook of Physical Constants, edited by T. J. Ahrens (American Geophysical Union Books Board, Washington, D.C., 1995), pp. 189-204.

[34] K. Karkkainen, A. Sihvola, and K. Nikoskinen, IEEE Trans. Geosci. Remote Sens. GE-39, 1013 (2001).

[35] J. M. Valverde, A. Castellanos, and M. A. S. Quintanilla, Contemp. Phys. 44, 389 (2003).

[36] J. M. Valverde, A. Castellanos, and M. A. S. Quintanilla, Phys. Rev. E 64, 021302 (2001).

[37] J. M. Valverde, A. Ramos, A. Castellanos, and P. K. Watson, Powder Technol. 97, 237 (1998).

[38] P. C. Carman, Trans. Inst. Chem. Eng. 15, 150 (1937).

[39] A. Castellanos, J. M. Valverde, and M. A. S. Quintanilla, Kona 22, 66 (2004).

[40] A. Castellanos, Adv. Phys. 54, 263 (2005).
[41] H. C. Hamaker, Physica (Amsterdam) 4, 1058 (1937).

[42] J. Visser, Adv. Colloid Interface Sci. 3, 331 (1972).

[43] S. Ross and I. D. Morrison, Colloidal Systems and Interfaces (Wiley-Interscience, New York, 1988).

[44] H. Rumpf, Chem.-Ing.-Tech. 30, 144 (1958).

[45] M. A. S. Quintanilla, A. Castellanos, and J. M. Valverde, Phys. Rev. E 64, 031301 (2001).

[46] M. Suzuki, K. Makino, M. Yamada, and K. Iinoya, Int. Chem. Eng. 21, 482 (1981).

[47] M. A. S. Quintanilla, J. M. Valverde, and A. Castellanos, AIChE J. 52, 1715 (2006).

[48] A. Castellanos, M. A. S. Quintanilla, J. M. Valverde, and C. Soria-Hoyo, Rev. Sci. Instrum. 78, 073901 (2007).

[49] C. Tan and T. B. Jones, J. Appl. Phys. 73, 3593 (1993).

[50] K. Taylor, P. J. King, and M. R. Swift, Phys. Rev. E 78, 031304 (2008).

[51] T. B. Jones, G. L. Whittaker, and T. J. Sulenski, Powder Technol. 49, 149 (1987)

[52] T. B. Jones, Electromechanics of Particles (Cambridge University Press, Cambridge, U.K., 1995).

[53] J. D. Jackson, Classical Electrodynamics (John Wiley \& Sons, New York, 1999).

[54] R. E. Rosensweig, G. R. Jerauld, and M. Zahn, Continuum Models of Discrete Systems, edited by O. Brulin and R. K. T. Hsieh (North-Holland, Amsterdam, 1981), pp. 137-144. 